\title{
Localized Molecular Orbitals in the Hückel Model of Perturbed Alternant Hydrocarbons and Their Relation to the Charge-Bond Order Matrix
}

\author{
V. GINEITYTE \\ Institute of Theoretical Physics and Astronomy, Vilnius University, Gostauto 12, LT-01108 Vilnius, \\ Lithuania
}

Received 10 January 2005; accepted 4 April 2005

Published online 20 June 2005 in Wiley InterScience (www.interscience.wiley.com). DOI 10.1002/qua.20689

\footnotetext{
ABSTRACT: An interrelation is expected between the charge-bond order matrix and the relevant representation matrix of noncanonical (localized) molecular orbitals (NCMOs) of perturbed alternant hydrocarbons (PAHs), as was the case with parent AHs. Accordingly, a single procedure is developed to obtain these matrices directly on the basis of solution of respective noncanonical one-electron problems in the framework of the simple Hückel model, i.e., of the commutation equation for the one-electron density matrix and of the block-diagonalization problem for the Hamiltonian matrix, the latter originating from the Brillouin theorem. The procedure consists of three principal steps: (i) an initial passing to the basis of NCMOs of parent AHs by means of the nonperturbative block diagonalization of the relevant common Hamiltonian matrix (H) represented in the basis of $2 p_{z}$ AOs of carbon atoms; followed by (ii) application of the noncommutative Rayleigh-Schrödinger perturbation theory to solve the abovespecified noncanonical problems; and (iii) subsequent retransformation of the power series obtained into the initial basis of $2 p_{z}$ AOs. Rederivation of the classical results concerning the charge and bond order redistributions in AHs due to perturbation (including the rule of the alternating polarity for one-center perturbations) following from the above-described procedure indicates that these results are actually part of the noncanonical theory of MOs for PAHs. The principal achievement of the study, however, consists of obtaining general algebraic expressions for the common NCMO representation matrix of PAHs in terms of submatrices (blocks) of the relevant chargebond order matrix. These expressions permit not only investigation of the effect of perturbation on the shapes of localized MOs, but also demonstrate the relationship between the given reshaping of these MOs and the respective charge redistribution. For local perturbations of a specific Coulomb parameter, reshaping of a single NCMO is shown to reflect the rule of the alternating polarity, namely of NCMO whose principal $\mathrm{AO}$ coincides with the site of perturbation. Moreover, the overall reshaping pattern of NCMOs was found in line with predictions of the simple resonance theory about
} 
increased contributions of certain quinoidal structures to the electronic structures of PAHs due to perturbations. NCMOs of pyridine and biphenyl molecules are studied in detail as examples. (C) 2005 Wiley Periodicals, Inc. Int J Quantum Chem 105: 232-245, 2005

Key words: alternant conjugated hydrocarbons; localized molecular orbitals; chargebond order matrix; pyridine; biphenyl

\section{Introduction}

$\mathbf{T}$ he chemical classification of molecules is known to be based on the presumed different extents of localization of electron pairs relevant to individual bonds in real space [1, 2]. In some cases, passing from one class of compounds to another is assumed to be accompanied by a stepwise and significant alteration in the extent of localization of electrons (cf. the formation of cyclic [aromatic] hydrocarbons from aliphatic ones [3]). Alternatively, changes in both structure and localization are considered small perturbations. This case may be exemplified by turning from aromatic hydrocarbons to their nitrogen-containing derivatives (heterocycles), i.e., from benzene to pyridine molecules [4].

Quantum chemical representation of the abovedescribed differences in localization, however, is met with considerable difficulties. Indeed, the unique standard (canonical) molecular orbitals (MOs) usually embrace the whole molecule under study, whatever its chemical structure (see, e.g., Ref. [5]), and, consequently, exhibit no substantial differences for different classes of compounds. In contrast, the alternative (noncanonical) MOs are not unique $[6,7]$. In this connection, various "external" localization criteria are usually invoked when looking for the so-called localized molecular orbitals (LMOs) [8]. When comparing molecules of different classes, however, the adequacy of a single localization criterion is hardly self-evident.

In this context, a direct method of obtaining noncanonical molecular orbitals (NCMOs) [9-17] on the basis of the Brillouin theorem [7, 8] deserves particular attention. The point is that invoking of "external" localization criteria may be avoided in this approach (e.g., by starting with a specific set of initially localized orbitals and determining their "tails" [10-12]). As a result, the actual extent of localization of NCMOs may be expected to depend only on the "internal" factors, including the chemical constitution of the given compound. Another attractive feature of the Brillouin theorem consists of its relation to the commutation equation for the relevant one-electron density matrix (DM) [13-15]. This, in turn, offers as an alternative method of looking for NCMOs that are related to the unique DM of the same system as closely as possible, and thereby allows us to remove the usual ambiguity in defining these orbitals. The resulting NCMOs and the interrelated charge-bond order matrix may then be considered as alternative noncanonical ways of describing electronic structures.

Among particular forms of the Billouin theorem, there is a zero value requirement for an off-diagonal element of the Fockian operator referring to an occupied and a vacant off-diagonal block (submatrix) of the total Fockian matrix in the basis of NCMOs being sought [10-16]. As a result, the block-diagonalization problem for the same matrix is obtained. The latter was shown to be solvable in the form of power series, provided that a zero-order matrix of a block-diagonal constitution may be revealed in the total Fockian (or Hamiltonian) matrix of the system(s) under study [13-17]. The same refers to the commutation equation for the oneelectron DM. In other terms, perturbative solutions of noncanonical one-electron problems proved to exist for systems representable by weakly interacting subsets of basis functions separated by substantial energy gaps. The approach used to obtain these solutions was referred to as the noncommutative Rayleigh-Schrödinger perturbation theory (NCRSPT) [16-18].

The above-described solution of the block-diagonalization problem in the form of power series has been applied primarily to saturated molecules [10$15,18-21]$, as well as to aliphatic conjugated hydrocarbons [22] in the basis of bond orbitals. As a result, LMOs of the bond-orbital-and-tail constitution have been obtained and analyzed. The principal achievements of these studies are as follows. First, conditions that ensure the very existence of LMOs of the above-specified type have been explored [10]. Second, more significant tails of LMOs, and thereby a larger extent of their delocalization, were found for aliphatic unsaturated hydrocarbons as compared with respective saturated analogues [22], in accordance with the expectation of the clas- 
sical chemistry. Finally, the inductive effect of heteroatom in nonaromatic hydrocarbons was shown to be interpretable in terms of LMOs that have changed shape relative to those of the parent system [20].

As opposed to aliphatic hydrocarbons, their aromatic analogues do not belong to systems representable by weakly-interacting subsets of basis functions. Quite the reverse, the so-called alternant hydrocarbons (that embrace the majority of the aromatic ones) are characterized by two strongly interacting subsets of $\mathrm{AOs}[3,7,22,23]$. That is why a nonperturbative solution of the block-diagonalization problem has been suggested for the common Hückel-type Hamiltonian matrix of alternant hydrocarbons, and the relevant NCMOs have been studied [24]. The results obtained allowed us to support the hypothesis of the classical chemistry about a stepwise increase of delocalization when passing from aliphatic hydrocarbons to aromatic ones, as well as to prove the interpretation of the relevant charge-bond order matrix as a part of the noncanonical theory of MOs.

Numerous organic compounds are known to be representable as perturbed alternant hydrocarbons (PAHs) [3]. This primarily refers to aromatic heterocycles (e.g., pyridine) that may be qualitatively described by small alterations in Coulomb parameters corresponding to the site(s) of substitution [4] (cf. the so-called one-center perturbations [3]). Moreover, breaking and/or formation of new bonds inside and/or between AHs may be represented accordingly by changes in resonance parameters. It is also worth mentioning that the PAHs are used as model systems when studying various aspects of chemical reactivity. To this end, the concept of topological equivalence of different molecular systems is invoked as well [3].

Rules governing the charge and bond order redistributions in AHs due to perturbation are among the classical results of quantum chemistry [3, 25$32]$. The rule of the alternating polarity $[26,32]$ for the case of a one-center perturbation may be mentioned as the most outstanding example. In our context, these rules are likely to build up a part of the noncanonical theory of perturbed AHs.

The aim of the present study is to verify the above-expressed expectation and to develop the remaining part of the same theory describing the effects of perturbations on the shapes of NCMOs of alternant hydrocarbons. To this end, we are about to formulate a general noncanonical formalism for PAHs embracing derivations of both the NCMO representation matrix and the related charge-bond order matrix directly without invoking the canonical MOs. On this basis, we will look for a relation between charge and bond-order redistributions due to perturbation, as well as the respective reshapings of NCMOs.

As was noted above, the common Hückel Hamiltonian matrix of AHs represented in the basis of $2 p_{z}$ AOs of carbon atoms may be transformed into a block-diagonal form [24]. This implies that a blockdiagonal zero-order term reveals itself in the total Hamiltonian matrix of perturbed AHs in the basis of NCMOs of parent hydrocarbons. As a result, the NCRSPT of Ref. [16] may be applied to solve the relevant block-diagonalization problem and the respective commutation equation that are expected to yield the NCMO representation matrix of PAHs in the basis of NCMOs of the parent AHs and the corresponding one-electron DM. To turn to the more convenient initial representations of the same characteristics, separate terms of the power series obtained should then be retransformed into the basis of $2 p_{z}$ AOs again. Precisely this scheme will be accomplished in this study.

We start with the most general formalism of the noncanonical theory for PAHs referring to perturbation matrix of an arbitrary structure. Thereupon, we specify the latter and analyze the resulting expressions for NCMOs. Finally, we will consider particular molecules as examples.

\section{General Formalism of the Noncanonical Theory of MOs for Perturbed AHs}

Let us begin with the simple Hückel model for AHs $[7,22,23,31]$. The basis set $\{\chi\}$ of any AH consisting of $2 p_{z}$ AOs of carbon atoms is known to be divisible in this model into two subsets $\left\{\chi_{1}\right\}$ and $\left\{\chi_{2}\right\}$ so that the intrasubset resonance parameters take zero values. Given that the Coulomb parameters $(\alpha)$ are also assumed to be uniform and the equality $\alpha=0$ is accepted for convenience, the model Hamiltonian matrices of AHs acquire a common form containing zero submatrices (blocks) in its diagonal positions:

$$
\mathbf{H}_{(0)}=\left|\begin{array}{cc}
\mathbf{0} & \mathbf{B} \\
\mathbf{B}^{+} & \mathbf{0}
\end{array}\right|,
$$

where $\mathbf{B}$ and $\mathbf{B}^{+}$are off-diagonal blocks containing intersubset resonance parameters. Nonzero values 
of the latter represent the neighboring pairs of AOs. The mean value of these resonance parameters $(\beta)$ will serve as a (negative) energy unit in our study; i.e., the equality $\beta=1$ will be accepted. Superscript + is used in Eq. (1) and below for transposed (Hermitian-conjugate) matrices. The Hamiltonian matrix of Eq. (1) will serve as the zero-order matrix of the perturbed AHs. That is why it is additionally supplied with subscript (0).

The expression for the unique charge-bond order matrix of AHs $\left(\mathbf{P}_{(0)}\right)$ was originaly derived by G. G. Hall [33] and rederived in Ref. [24] on the basis of solution of the commutation equation for the one-electron DM. Two alternative forms of this expression have been obtained:

$$
\mathbf{P}_{(0)}=\left|\begin{array}{cc}
\mathbf{I} & \mathbf{R B} \\
\mathbf{B}^{+} \mathbf{R} & \mathbf{I}
\end{array}\right|, \quad \mathbf{P}_{(0)}=\left|\begin{array}{cc}
\mathbf{I} & \mathbf{B Q} \\
\mathbf{Q B} & \mathbf{I}
\end{array}\right|,
$$

where

$$
\begin{gathered}
\mathbf{R}=\left(\mathbf{B B}^{+}\right)^{-1 / 2}, \quad \mathbf{Q}=\left(\mathbf{B}^{+} \mathbf{B}\right)^{-1 / 2}, \\
\mathbf{R} \mathbf{B}=\mathbf{B} \mathbf{Q}
\end{gathered}
$$

and I here and below stands for the unit matrix.

The block-diagonalization transformation for the same matrix $\mathbf{H}_{(0)}$ takes the form

$$
\tilde{\mathbf{H}}_{(0)}=\mathbf{C}^{+} \mathbf{H}_{(0)} \mathbf{C}=\left|\begin{array}{cc}
\mathbf{E}_{(0) 1} & \mathbf{0} \\
\mathbf{0} & -\mathbf{E}_{(0) 2}
\end{array}\right|,
$$

where $\mathbf{C}$ is a unitary matrix [24] containing the vectors of coefficients of NCMOs being sought in its columns, and $\mathbf{E}_{(0) 1}$ and $-\mathbf{E}_{(0) 2}$ are the so-called eigenblocks of the matrix $\mathbf{H}_{(0)}$. [The minus sign in front of $\mathbf{E}_{(0) 2}$ is introduced for convenience.]

As may be expected on the basis of ambiguity in determining NCMOs in general (Section 1), the transformation matrix $\mathbf{C}$ of Eq. (5) is not unique. The criterion underlying the choice of this matrix made in Ref. [24] consists of maximal similarity of its constitution to that of the charge-bond order matrix $\mathbf{P}_{(0)}$ of Eq. (2). The resulting matrix $\mathbf{C}$ has been alternatively expressed as follows:

$$
\mathbf{C}=\frac{1}{\sqrt{2}}\left|\begin{array}{cc}
\mathbf{I} & \mathbf{R B} \\
\mathbf{B}^{+} \mathbf{R} & -\mathbf{I}
\end{array}\right|, \quad \mathbf{C}=\frac{1}{\sqrt{2}}\left|\begin{array}{cc}
\mathbf{I} & \mathbf{B Q} \\
\mathbf{Q B}^{+} & -\mathbf{I}
\end{array}\right| .
$$

The relevant formulae for the eigenblocks of the matrix $\mathbf{H}_{(0)}$ take the form

$$
\mathbf{E}_{(0) 1}=\mathbf{R}^{-1}=\left(\mathbf{B B}^{+}\right)^{1 / 2}, \quad \mathbf{E}_{(0) 2}=\mathbf{Q}^{-1}=\left(\mathbf{B}^{+} \mathbf{B}\right)^{1 / 2}
$$

Finally, the unitarity condition for the matrix C yields the following useful relations:

$$
\mathbf{R B B}^{+} \mathbf{R}=\mathbf{Q B}^{+} \mathbf{B Q}=\mathbf{B Q Q B}^{+}=\mathrm{BRRB}^{+}=\mathbf{I},
$$

where Eq. (4) is also invoked. In addition, it should be noted that proportionality between separate submatrices of matrices $\mathbf{P}_{(0)}$ and $\mathbf{C}$ seen from comparison of Eqs. (2) and (6) ensures the actual coincidence between vectors of coefficients of occupied NCMOs of AHs and the respective columns (rows) of the charge-bond order matrix (up to the normalization factor $1 / \sqrt{2}$ ).

Let us turn now to the case of perturbed AHs. The respective first-order (perturbation) matrix $\mathbf{H}_{(1)}$ may also be divided accordingly into separate submatrices:

$$
\mathbf{H}_{(1)}=\left|\begin{array}{cc}
\mathbf{A}_{(1)} & \mathbf{W}_{(1)} \\
\mathbf{W}_{(1)}^{+} & \mathbf{D}_{(1)}
\end{array}\right| .
$$

No specification of these submatrices is undertaken in this section. It is evident that the first-order matrix $\mathbf{H}_{(1)}$ of Eq. (9) may be transformed into the basis of NCMOs of parent hydrocarbons. As a result, the total transformed Hamiltonian matrix

$$
\tilde{\mathbf{H}}=\mathbf{C}^{+} \mathbf{H C}=\mathbf{C}^{+} \mathbf{H}_{(0)} \mathbf{C}+\mathbf{C}^{+} \mathbf{H}_{(1)} \mathbf{C}=\tilde{\mathbf{H}}_{(0)}+\tilde{\mathbf{H}}_{(1)}
$$

complies with the requirements of the NCRSPT; i.e., it contains a block-diagonal zero-order term $\tilde{\mathbf{H}}_{(0)}$ of Eq. (5). For matrices of this particular constitution, both the block-diagonalization problem and the commutation equation for the relevant DM $\tilde{\mathbf{P}}$ have been analyzed in Ref. [15]. Thus, the matrix $\mathbf{T}$ derived in Ref. [15] may be invoked to transform the total matrix $\tilde{\mathbf{H}}$ into a block-diagonal form. We then obtain

$$
\tilde{\mathbf{H}}^{\prime}=\mathbf{T}^{+} \tilde{\mathbf{H}} \mathbf{T}=\left|\begin{array}{cc}
\mathbf{E}_{1} & \mathbf{0} \\
\mathbf{0} & -\mathbf{E}_{2}
\end{array}\right|,
$$

where $\mathbf{E}_{1}$ and $-\mathbf{E}_{2}$ are the eigenblocks of the total Hamiltonian matrix of perturbed AHs. The unitary matrix T of Ref. [15] consists of a sum of corrections $\mathbf{T}_{(k)}$ of various orders $(k)$. The zero-order term of this series coincides with the unit matrix $\left[\mathbf{T}_{(0)}=\mathbf{I}\right]$, 
while the first-order correction $\mathbf{T}_{(1)}$ has been chosen to resemble the relevant unique correction $\tilde{\mathbf{P}}_{(1)}$ as closely as possible. As a result, proportionality between separate submatrices of matrices $\tilde{\mathbf{P}}_{(1)}$ and $\mathbf{T}_{(1)}$ has been achieved:

$$
\tilde{\mathbf{P}}_{(1)}=-2\left|\begin{array}{cc}
\mathbf{0} & \mathbf{G}_{(1)} \\
\mathbf{G}_{(1)}^{+} & \mathbf{0}
\end{array}\right|, \quad \mathbf{T}_{(1)}=\left|\begin{array}{cc}
\mathbf{0} & \mathbf{G}_{(1)} \\
-\mathbf{G}_{(1)}^{+} & \mathbf{0}
\end{array}\right|,
$$

where the common principal matrix $\mathbf{G}_{(1)}$ of both expressions meets the equation

$$
\mathbf{G}_{(1)} \mathbf{Q}+\mathbf{R G}_{(1)}+\mathbf{R} \tilde{\mathbf{H}}_{(1) 12} \mathbf{Q}=\mathbf{0},
$$

and $\tilde{\mathbf{H}}_{(1) 12}$ stands for the off-diagonal block of the first-order matrix $\tilde{\mathbf{H}}_{(1)}$ defined by Eq. (10). The expressions for eigenblocks $\mathbf{E}_{(0) 1}$ and $\mathbf{E}_{(0) 2}$ in terms of matrices $\mathbf{R}$ and $\mathbf{Q}$ shown in Eq. (7) are also used when deriving Eq. (13).

Now, no more is required than to go back to the initial basis of $2 p_{z}$ AOs $\{\chi\}$. Retransformation of the correction $\left[\tilde{\mathbf{P}}_{(1)}\right]$ to the charge-bond order matrix yields

$$
\mathbf{P}_{(1)}=\mathbf{C} \tilde{\mathbf{P}}_{(1)} \mathbf{C}^{+}=\left|\begin{array}{cc}
\mathbf{X}_{(1)} & \mathbf{N}_{(1)} \\
\mathbf{N}_{(1)}^{+} & \mathbf{Z}_{(1)}
\end{array}\right|
$$

The diagonal blocks of correction $\mathbf{P}_{(1)}$ are Hermitian (symmetric) matrices:

$$
\begin{aligned}
\mathbf{X}_{(1)}=-\mathbf{B Q G}_{(1)}^{+}-\mathbf{G}_{(1)} \mathbf{Q B}^{+}, \\
\mathbf{Z}_{(1)}=\mathbf{G}_{(1)}^{+} \mathbf{B} \mathbf{Q}+\mathbf{Q B} \mathbf{B}_{(1),}
\end{aligned}
$$

while the off-diagonal block $\mathbf{N}_{(1)}$ may be alternatively expressed as

$$
\mathbf{N}_{(1)}=-\mathbf{B Q} \boldsymbol{\Delta}_{(1)}=-\boldsymbol{\Gamma}_{(1)} \mathbf{B Q},
$$

where

$$
\begin{aligned}
& \Delta_{(1)}=\mathbf{G}_{(1)}^{+} \mathbf{B Q}-\mathbf{Q B}^{+} \mathbf{G}_{(1)}, \\
& \Gamma_{(1)}=\mathbf{B Q G}_{(1)}^{+}-\mathbf{G}_{(1)} \mathbf{Q B}^{+}
\end{aligned}
$$

are skew-Hermitian (skew-symmetric) matrices. Finally, the following useful relations between the above-introduced matrices

$$
\mathbf{X}_{(1)}=-\mathbf{B} \mathbf{Q Z} \mathbf{Z}_{(1)} \mathbf{Q B}^{+}, \quad \boldsymbol{\Gamma}_{(1)}=\mathbf{B} \mathbf{Q} \boldsymbol{\Delta}_{(1)} \mathbf{Q B}^{+}
$$

deserve mention. It is evident that elements of the matrix $\mathbf{P}_{(1)}$ of Eqs. (14)-(17) represent the charge and bond order redistribution in AHs due to perturbation.

Again, the final NCMO representation matrix for perturbed AHs $(\mathbf{U})$ coincides with the total transformation matrix of the initial Hamiltonian matrix $\mathbf{H}$ into the block-diagonal form. To derive the relevant expression, the product of $\mathbf{C}^{+} \mathbf{H C}$ should be substituted for $\tilde{\mathbf{H}}$ into Eq. (11) in accordance with Eq. (10). We then obtain

$$
\mathbf{U}=\mathbf{C T}=\mathbf{C}+\mathbf{C} \mathbf{T}_{(1)}+\mathbf{C T}_{(2)}+\ldots,
$$

where equality $\mathbf{T}_{(0)}=\mathbf{I}[15]$ is used. Matrix $\mathbf{U}$ evidently contains vectors of the coefficients of NCMOs of PAHs represented in the basis of AOs $\{\chi\}$ in its separate columns. It is also seen that zeroorder NCMOs of PAHs coincide with those of parent $\mathrm{AHs}$, whereas their reshaping pattern due to perturbation is expected to be described mainly by the first-order correction $\mathbf{U}_{(1)}$ of the power series for matrix $\mathbf{U}$. The expression for this correction easily follows from Eqs. (6), (12), and (19):

$$
\mathbf{U}_{(1)}=\mathbf{C T}_{(1)}=\frac{1}{\sqrt{2}}\left|\begin{array}{cc}
-\mathbf{B Q G}_{(1)}^{+} & \mathbf{G}_{(1)} \\
\mathbf{G}_{(1)}^{+} & \mathbf{Q B}^{+} \mathbf{G}_{(1)}
\end{array}\right|,
$$

where the $\mathbf{Q}$ representation of the matrix $\mathbf{C}$ is used.

Comparison of Eqs. (14)-(17) and (20) shows that similar matrix products (e.g., $\mathbf{B Q G}_{(1)}^{+}$) arise within first-order matrices $\mathbf{U}_{(1)}$ and $\mathbf{P}_{(1)}$. The overall extent of their similarity, however, is reduced versus that observed when comparing the matrices of Eqs. (2) and (6), as well as those of Eq. (12). As a result, no coincidence may be generally expected between vectors of coefficients of NCMOs of PAHs and the respective columns (rows) of the relevant chargebond order matrix in the basis of AOs. Nevertheless, the localized nature of NCMOs [24] remains (see Section 4).

Before completing this discussion, let us dwell on the relationship between the above-obtained corrections for the charge-bond order matrix of PAHs and the classical perturbation theory in the Hückel method in terms of various types of polarizabilities (e.g., atom-atom, atom-bond) [4, 25-31]. The latter are known to be definable in terms of derivatives of particular elements of the chargebond order matrix of the perturbed system with respect to separate elements of matrix $\mathbf{H}_{(1)}[4,30]$. The zero-order contribution of our series $\left(\mathbf{P}_{(0)}\right)$ evidently yields no increments to these derivatives. 
Hence, the analogues of the classical polarizabilities take the form

$$
\Pi_{i, j}^{(11)}=\frac{\partial \mathbf{X}_{(1) i i}}{\partial \mathbf{A}_{(1) j j}}, \quad \Pi_{k, j}^{(21)}=\frac{\partial \mathbf{Z}_{(1) k k}}{\partial \mathbf{A}_{(1) j j}}, \quad \Pi_{i k, j}=\frac{\partial \mathbf{N}_{(1) i k}}{\partial \mathbf{A}_{(1) j j}}, \text { etc., }
$$

where $\Pi_{i, j}^{(11)}$ and $\Pi_{k, j}^{(21)}$ are atom-atom polarizabilities for pairs of AOs (atoms) of the first subset and for those of different subsets, respectively, and $\Pi_{i k, j}$ is an example of polarizability of the type bond atom.

\section{Consideration of Specific Perturbation Matrices}

\subsection{BLOCK-DIAgONAL PERTURBATION MATRIX}

Let us begin with the block-diagonal perturbation matrix defined as

$$
\mathbf{H}_{(1)}^{(\alpha)}=\left|\begin{array}{cc}
\mathbf{A}_{(1)} & \mathbf{0} \\
\mathbf{0} & \mathbf{D}_{(1)}
\end{array}\right|,
$$

and corresponding to case $\mathbf{W}_{(1)}=\mathbf{0}$ in Eq. (9). Inasmuch as this matrix embraces the most popular local perturbation of the Coulomb parameter, it is supplied with the superscript $(\alpha)$. The relevant offdiagonal block $\tilde{\mathbf{H}}_{(1) 12}^{(\alpha)}$ contained within the principal matrix equation of Eq. (13) takes the form

$$
\tilde{\mathbf{H}}_{(1) 12}^{(\alpha)}=\frac{1}{2}\left[\mathbf{A}_{(1)} \mathbf{B Q}-\mathbf{B Q} \mathbf{D}_{(1)}\right] .
$$

Use of these particular expressions within Eqs. (14)-(17) and (20) allows us to derive the respective matrices $\mathbf{P}_{(1)}^{(\alpha)}$ and $\mathbf{U}_{(1)}^{(\alpha)}$ and to study their specific interrelations. Before doing this, however, we will consider the properties of the respective principal matrix $\mathbf{G}_{(1)}^{(\alpha)}$ defined by Eqs. (13) and (23).

Let us begin with the proof of the following relations:

$$
\mathbf{G}_{(1)}^{(\alpha)+} \mathbf{B} \mathbf{Q}=\mathbf{Q B}^{+} \mathbf{G}_{(1)}^{(\alpha)}, \quad \mathbf{B Q G}_{(1)}^{(\alpha)+}=\mathbf{G}_{(1)}^{(\alpha)} \mathbf{Q B}^{+}
$$

The initial stage of this proof consists of eliminating the matrix $\mathbf{R}$ from Eq. (13) by multiplying this relation by $\mathbf{B}^{+}$from the left-hand side and replacing the product $\mathbf{B}^{+} \mathbf{R}$ by $\mathbf{Q B}^{+}$in accordance with Eq. (4). We then obtain

$$
\mathbf{B}^{+} \mathbf{G}_{(1)}^{(\alpha)} \mathbf{Q}+\mathbf{Q B}^{+} \mathbf{G}_{(1)}^{(\alpha)}+\mathbf{Q B}^{+} \tilde{\mathbf{H}}_{(1) 12}^{(\alpha)} \mathbf{Q}=\mathbf{0}
$$

Let Eq. (25) be subsequently multiplied by $\mathbf{Q}$ from the left-hand side. Accordingly, the complexconjugate counterpart of the same relation will be multiplied by $\mathbf{Q}$ from the right-hand side. After subtracting the resulting relations and invoking the expression for $\tilde{\mathbf{H}}_{(1) 12}^{(\alpha)}$ of Eq. (23), we obtain

$$
\begin{aligned}
& \mathbf{Q}\left[\mathbf{G}_{(1)}^{(\alpha)+} \mathbf{B} \mathbf{Q}-\mathbf{Q B}^{+} \mathbf{G}_{(1)}^{(\alpha)}\right] \\
&+\left[\mathbf{G}_{(1)}^{(\alpha)+} \mathbf{B} \mathbf{Q}-\mathbf{Q B}^{+} \mathbf{G}_{(1)}^{(\alpha)}\right] \mathbf{Q}=\mathbf{0} .
\end{aligned}
$$

The relation of Eq. (26) coincides with the specific case of matrix equations of the form $\mathbf{A X}+\mathbf{X} \mathbf{B}+$ $\mathbf{C}=\mathbf{0}$ [34], where $\mathbf{C}=\mathbf{0}$. The latter equality is known to yield a zero solution of these equations $(\mathbf{X}=\mathbf{0})$. Thus, coincidence of the total matrix witin the braces of Eq. (26) to the zero matrix follows, and thereby the first relation of Eq. (24) is obtained. By multiplying the latter by $\mathbf{B} \mathbf{Q}$ and $\mathbf{Q B}^{+}$from its leftand right-hand sides, respectively, and by the subsequent use of Eq. (8), the second result of Eq. (24) may easily be derived.

The principal relations of Eq. (24) have important consequences. In particular, equalities $\Delta_{(1)}^{(\alpha)}=\boldsymbol{\Gamma}_{(1)}^{(\alpha)}=$ $\mathbf{0}$ and thereby $\mathbf{N}_{(1)}^{(\alpha)}=\mathbf{0}$ follow immediately from Eqs. (16), (17), and (24). Consequently, the firstorder correction $\mathbf{P}_{(1)}^{(\alpha)}$ takes the form

$$
\mathbf{P}_{(1)}^{(\alpha)}=\left|\begin{array}{cc}
\mathbf{X}_{(1)}^{(\alpha)} & \mathbf{0} \\
\mathbf{0} & \mathbf{Z}_{(1)}^{(\alpha)}
\end{array}\right|
$$

It is seen that intersubset bond orders are not influenced by the perturbation $\mathbf{H}_{(1)}^{(\alpha)}$ of Eq. (22). This result may be considered as the matrix generalization of the classical conclusion regarding zero values of polarizabilities of the type of bond atom for AHs $[26,27,30]$, the latter being defined by the last relation of Eq. (21).

Furthermore, use of Eq. (24) within the definitions of matrices $\mathbf{X}_{(1)}^{(\alpha)}$ and $\mathbf{Z}_{(1)}^{(\alpha)}$ shown in Eq. (15) yields the following expressions for the latter:

$$
\begin{aligned}
& \mathbf{X}_{(1)}^{(\alpha)}=-2 \mathbf{B} \mathbf{Q} \mathbf{G}_{(1)}^{(\alpha)+}=-2 \mathbf{G}_{(1)}^{(\alpha)} \mathbf{Q B} \mathbf{B}^{+}, \\
& \mathbf{Z}_{(1)}^{(\alpha)}=2 \mathbf{G}_{(1)}^{(\alpha)+} \mathbf{B Q}=2 \mathbf{Q B} \mathbf{B}^{+} \mathbf{G}_{(1)}^{(\alpha)} .
\end{aligned}
$$

Finally, Eqs. (8) and (28) yield the following alternative formulae for the matrix $\mathbf{G}_{(1)}^{(\alpha)}$ :

$$
\mathbf{G}_{(1)}^{(\alpha)}=\frac{1}{2} \mathbf{B} \mathbf{Q} \mathbf{Z}_{(1)}^{(\alpha)}=-\frac{1}{2} \mathbf{X}_{(1)}^{(\alpha)} \mathbf{B} \mathbf{Q}
$$


Use of Eq. (29) within Eq. (20) allows the first-order correction $\mathbf{U}_{(1)}^{(\alpha)}$ to the NCMO representation matrix to be expressed in terms of matrices $\mathbf{X}_{(1)}^{(\alpha)}$ and $\mathbf{Z}_{(1)}^{(\alpha)}$ :

$$
\mathbf{U}_{(1)}^{(\alpha)}=\frac{1}{2 \sqrt{2}}\left|\begin{array}{cc}
\mathbf{X}_{(1)}^{(\alpha)} \mathbf{Q B} \mathbf{B}^{+} & -\mathbf{X}_{(1)}^{(\alpha)} \mathbf{B} \mathbf{Q} \\
\mathbf{Z}_{(1)}^{(\alpha)}
\end{array}\right| .
$$

It is seen that nonzero off-diagonal blocks are present in the correction $\mathbf{U}_{(1)}^{(\alpha)}$, in contrast to $\mathbf{P}_{(1)}^{(\alpha)}$ of Eq. (27).

The final step of this study consists of the derivation of equations for matrices $\boldsymbol{X}_{(1)}^{(\alpha)}$ and $\mathbf{Z}_{(1)}^{(\alpha)}$. The definitions of these matrices shown in Eq. (15) differ from those for matrices $\boldsymbol{\Delta}_{(1)}$ and $\boldsymbol{\Gamma}_{(1)}$ of Eq. (17) only in signs of some matrix products. For example, the expression for $\mathbf{Z}_{(1)}$ differs from that for $\boldsymbol{\Delta}_{(1)}$ only in the sign in front of the product $\mathbf{Q B} \mathbf{B}^{+} \mathbf{G}_{(1)}$. Thus, the procedure like that used when deriving Eq. (26) should be performed when looking for the aboveexpected equations, only subtracting of relations should be replaced by their summation. The result is as follows:

$$
\mathbf{Q} \mathbf{Z}_{(1)}^{(\alpha)}+\mathbf{Z}_{(1)}^{(\alpha)} \mathbf{Q}+\left[\mathbf{Q}^{2} \mathbf{B}^{+} \mathbf{A}_{(1)} \mathbf{B} \mathbf{Q}^{2}-\mathbf{Q D}_{(1)} \mathbf{Q}\right]=\mathbf{0}
$$

and belongs to matrix problems of the above-cited form ( $\mathbf{A X}+\mathbf{X B}+\mathbf{C}=\mathbf{0})$ [34], where $\mathbf{C}$ coincides now with the total matrix within the brackets. The solution may then be represented in the form

$\mathbf{Z}_{(1)}^{(\alpha)}=-\int_{0}^{\infty} e^{-\mathbf{Q}^{t}}\left[\mathbf{Q}^{2} \mathbf{B}^{+} \mathbf{A}_{(1)} \mathbf{B} \mathbf{Q}^{2}-\mathbf{Q D}_{(1)} \mathbf{Q}\right] e^{-\mathbf{Q} t} d t$

Note that $\mathbf{Q}$ is assumed to be a positive-definite matrix [24], and the relation of Eq. (31) is multiplied by -1 to obtain a negative-definite matrix required for expressing the solution in the form of an integral. Given that matrix $\mathbf{Z}_{(1)}^{(\alpha)}$ is obtained, the relevant matrix $\boldsymbol{X}_{(1)}^{(\alpha)}$ follows from Eq. (18).

The integral of Eq. (32) easily yields the famous rule of the alternating polarity [26, 29-32]. Indeed, the expressions for particular elements $\mathbf{Z}_{(1) k l}^{(\alpha)}$ following from Eq. (32) contain additive increments of separate elements $\mathbf{A}_{(1) i j}$ and $\mathbf{D}_{(1) m n}$. As a result, each derivative of Eq. (21), and thereby the relevant polarizability may be found independently. For the intersubset polarizability of the atom-atom type $\Pi_{k, j}^{(21)}$, a negative sign results immediately from Eq. (32):

$$
\Pi_{k, j}^{(21)}=\frac{\partial \mathbf{Z}_{(1) k k}^{(\alpha)}}{\partial \mathbf{A}_{(1) j j}}=-\int_{0}^{\infty}\left[\left(\mathbf{B} \mathbf{Q}^{2} e^{-\mathbf{Q} t}\right)_{j k}\right]^{2} d t<0 .
$$

Thus, the population of any $\mathrm{AO}\left(\chi_{k}\right)$ of the second subset is reduced after introducing a more electronegative heteroatom into the $j$ th position of the first subset $\left(\mathbf{A}_{(1) j j}>0\right.$ in our negative energy units in this case). For the intrasubset polarizability $\Pi_{i, j}^{(11)}$ of Eq. (21), a positive sign may be established similarly after invoking Eq. (18); accordingly, an increased population of any $\mathrm{AO}$ of the same subset is obtained. These results are nothing more than the rule of the alternating polarity.

Before finishing this subsection, let us note that application of the matrix $\mathbf{H}_{(1)}^{(\alpha)}$ of Eq. (22) is not restricted to modeling of substitution of carbon atoms by heteroatoms: This matrix may be also used to represent passing to more sophisticated models of hydrocarbons themselves, wherein resonance parameters between all pairs of AOs are explicitly included along with allowance for first-order alterations in Coulomb parameters. Indeed, resonance parameters between nonneighboring pairs of $\mathrm{AOs}$ of the intersubset type may be successfully incorporated into the zero-order matrix $\mathbf{H}_{(0)}$ of Eq. (1). (Application of the latter is not restricted to taking into account resonance parameters between neighboring pairs of AOs only.) Accordingly, the additional parameters of the intrasubset type may be included in the perturbation matrix $\mathbf{H}_{(1)}^{(\alpha)}$, the latter then describing the deviation of the actual model from that of an alternant hydrocarbon. This, in turn, implies that the results of this section are applicable to represent consequences of the above-specified extension of the model. In particular, Eq. (27) then indicates a certain invariance of the intersubset bond orders with respect to taking into account the additional parameters of the intrasubset type.

\subsection{ANTI-BLOCK-DIAGONAL PERTURBATION MATRIX} form

Let us now turn to the perturbation matrix of the

$$
\mathbf{H}_{(1)}^{(\beta)}=\left|\begin{array}{cc}
\mathbf{0} & \mathbf{W}_{(1)} \\
\mathbf{W}_{(1)}^{+} & \mathbf{0}
\end{array}\right|
$$

representing alterations in resonance parameters of the intersubset type. The relevant perturbed system evidently remains one of alternant hydrocarbons. 
Analysis of expressions for respective corrections $\mathbf{U}_{(1)}^{(\beta)}$ and $\mathbf{P}_{(1)}^{(\beta)}$ resembles that of the previous section. Thus, the new block $\tilde{\mathbf{H}}_{(1) 12}^{(\beta)}$ takes the form

$$
\tilde{\mathbf{H}}_{(1) 12}^{(\beta)}=\frac{1}{2} \mathbf{B} \mathbf{Q}\left[\mathbf{W}_{(1)}^{+} \mathbf{B} \mathbf{Q}-\mathbf{Q} \mathbf{B}^{+} \mathbf{W}_{(1)}\right]
$$

After substituting this expression into the matrix equation for $\mathbf{G}_{(1)}^{(\beta)}$ like that of Eq. (13), and performing an analogous procedure, we obtain the relations

$$
\mathbf{B Q G}_{(1)}^{(\beta)+}=-\mathbf{G}_{(1)}^{(\beta)} \mathbf{Q B}^{+}, \quad \mathbf{G}_{(1)}^{(\beta)+} \mathbf{B} \mathbf{Q}=-\mathbf{Q B}^{+} \mathbf{G}_{(1)}^{(\beta)}
$$

instead of those shown in Eq. (24). As a result, zero matrices stand for $\mathbf{Z}_{(1)}^{(\beta)}$ and for $\mathbf{X}_{(1)}^{(\beta)}$ in the new correction $\mathbf{P}_{(1)}^{(\beta)}$ :

$$
\mathbf{P}_{(1)}^{(\beta)}=\left|\begin{array}{cc}
\mathbf{0} & -\mathbf{B Q} \boldsymbol{\Delta}_{(1)}^{(\beta)} \\
\boldsymbol{\Delta}_{(1)}^{(\beta)} \mathbf{Q B}^{+} & \mathbf{0}
\end{array}\right|=\left|\begin{array}{cc}
\mathbf{0} & -\boldsymbol{\Gamma}_{(1)}^{(\beta)} \mathbf{B Q} \\
\mathbf{Q B}^{+} \boldsymbol{\Gamma}_{(1)}^{(\beta)} & \mathbf{0}
\end{array}\right| .
$$

Again, the interrelated matrices $\boldsymbol{\Delta}_{(1)}^{(\beta)}$ and $\boldsymbol{\Gamma}_{(1)}^{(\beta)}$ (see Eq. (18)) meet matrix equations of the above-considered form, e.g.,

$\mathbf{Q} \boldsymbol{\Delta}_{(1)}^{(\beta)}+\boldsymbol{\Delta}_{(1)}^{(\beta)} \mathbf{Q}+\mathbf{Q}\left(\mathbf{Q B} \mathbf{B}^{+} \mathbf{W}_{(1)}-\mathbf{W}_{(1)}^{+} \mathbf{B} \mathbf{Q}\right) \mathbf{Q}=\mathbf{0}$,

and thereby these are expressible in the form of integrals like that of Eq. (32). From Eq. (37), it follows that no intrasubset corrections arise in the matrix $\mathbf{P}_{(1)}^{(\beta)}$, in accordance with the alternant nature of the perturbed system. The result of Eq. (37) may be alternatively regarded as the matrix generalization of the classical conclusion regarding zero values for polarizabilities of the type of atom bond in AHs [26, 27, 30].

For the correction $\mathbf{U}_{(1)}^{(\beta)}$ to the NCMO representation matrix $\mathbf{U}^{(\beta)}$, we obtain

$$
\mathbf{U}_{(1)}^{(\beta)}=\frac{1}{2 \sqrt{2}}\left|\begin{array}{cc}
-\boldsymbol{\Gamma}_{(1)}^{(\beta)} & -\mathbf{B Q} \mathbf{\Delta}_{(1)}^{(\beta)} \\
\mathbf{Q B} \mathbf{B}^{+} \boldsymbol{\Gamma}_{(1)}^{(\beta)} & -\boldsymbol{\Delta}_{(1)}^{(\beta)}
\end{array}\right| .
$$

It is seen that nonzero contributions of the intrasubset type emerge within matrix $\mathbf{U}_{(1)}^{(\beta)}$. This implies that the diagonal blocks of the total NCMO representation matrix $\mathbf{C}+\mathbf{U}_{(1)}^{(\beta)}$ are no longer proportional to unit matrices, as is the case with matrix $\mathbf{C}$ of Eq. (6) referring to any alternant system. It may easily be shown, however, that zero matrices may be restored in the diagonal positions of the correction under study by turning to an alternative set of
NCMOs. Indeed, the total Hamiltonian matrix $\tilde{\mathbf{H}}^{\prime}$ of Eq. (11) remains of a block-diagonal structure after applying an additional unitary transformation of the same constitution:

$$
\tilde{\mathbf{H}}^{\prime \prime}=\mathbf{M}^{+} \tilde{\mathbf{H}}^{\prime} \mathbf{M}=\mathbf{M}^{+} \mathbf{T}^{+} \tilde{\mathbf{H}} \mathbf{T} \mathbf{M}=\left|\begin{array}{cc}
\mathbf{E}_{1}^{\prime} & \mathbf{0} \\
\mathbf{0} & -\mathbf{E}_{2}^{\prime}
\end{array}\right|,
$$

where

$$
\mathbf{M}=\left|\begin{array}{cc}
\mathbf{M}_{1} & \mathbf{0} \\
\mathbf{0} & \mathbf{M}_{2}
\end{array}\right|, \quad \mathbf{M}_{1} \mathbf{M}_{1}^{+}=\mathbf{I}, \quad \mathbf{M}_{2} \mathbf{M}_{2}^{+}=\mathbf{I}
$$

and $\mathbf{M}_{1}$ and $\mathbf{M}_{2}$ are arbitrary unitary submatrices. As a result, the matrix

$$
\mathbf{U}^{\prime}=\mathbf{C T M}=\mathbf{U M}
$$

is also an NCMO representation matrix. Using this rather self-evident result as a basis, we will then look for blocks $\mathbf{M}_{1}$ and $\mathbf{M}_{2}$ such that the diagonal blocks of the new matrix $\mathbf{U}^{\prime}$ coincide with matrices $(1 / \sqrt{2}) \mathbf{I}$ and $-(1 / \sqrt{2}) \mathbf{I}$, as was the case with matrix $\mathrm{C}$ of Eq. (6). This condition is resolved into following requirements:

$$
\left(\mathbf{I}-\frac{1}{2} \boldsymbol{\Gamma}_{(1)}^{(\beta)}\right) \mathbf{M}_{1}=\mathbf{I}, \quad-\left(\mathbf{I}+\frac{1}{2} \boldsymbol{\Delta}_{(1)}^{(\beta)}\right) \mathbf{M}_{2}=-\mathbf{I},
$$

which yield the solution of the form

$$
\mathbf{M}_{1}=\mathbf{I}+\frac{1}{2} \boldsymbol{\Gamma}_{(1)}^{(\beta)}, \quad \mathbf{M}_{2}=\mathbf{I}-\frac{1}{2} \boldsymbol{\Delta}_{(1)}^{(\beta)} .
$$

At the same time, the skew-symmetric (skew-Hermitian) nature of the first-order matrices $\boldsymbol{\Gamma}_{(1)}^{(\beta)}$ and $\boldsymbol{\Delta}_{(1)}^{(\beta)}$ ensures unitarity of submatrices $\mathbf{M}_{1}$ and $\mathbf{M}_{2}$. [It is worth mentioning that an analogous procedure fails for the case of the block-diagonal perturbation matrix of Eq. (22) just because of symmetric (Hermitian) nature of matrices $\mathbf{X}_{(1)}^{(\beta)}$ and $\mathbf{Z}_{(1)}^{(\beta)}$.] The offdiagonal blocks of the new matrix $\mathbf{U}^{(\beta)}$ ' may easily be obtained from Eqs. (6), (39), (41), (42), and (44). The final result takes the form

$$
\mathbf{U}^{(\beta) \prime}=\frac{1}{\sqrt{2}}\left|\begin{array}{cc}
\mathbf{I} & \mathbf{B Q}\left[\mathbf{I}-\boldsymbol{\Delta}_{(1)}^{(\beta)}\right] \\
\mathbf{Q B}^{+}\left[\mathbf{I}+\boldsymbol{\Gamma}_{(1)}^{(\beta)}\right] & -\mathbf{I}
\end{array}\right| .
$$

The new NCMO representation matrix $\mathbf{U}^{(\beta) \text { ' }}$ resembles the sum of matrices $\mathbf{P}_{(0)}$ and $\mathbf{P}_{(1)}^{(\beta)}$.

Let us now consider the case of an anti-blockdiagonal matrix of Eq. (34) that describes the formation of weak intermolecular bond(s) between 
initially isolated AHs. The new bond(s) are assumed to be representable by resonance parameter(s) of the first-order magnitude. For simplicity, let us confine ourselves to two identical AHs, I and II (cf. the formation of biphenyl by bonding of two benzene molecules [3]). The subsets of AOs of the total system $\left(\left\{\chi_{1}\right\}\right.$ and $\left.\left\{\chi_{2}\right\}\right)$ may be conveniently subdivided in this case into parts that refer to separate parent $\mathrm{AHs}$, e.g., the subset $\left\{\chi_{1}\right\}$ will consist of two parts $\left\{\chi_{1, I}\right\}$ and $\left\{\chi_{1, I I}\right\}$. As a result, the relevant total matrices $\mathbf{B}$ and $\mathbf{Q}$ of Eqs. (1) and (3) will contain nonzero submatrices of lower dimensions:

$$
\mathbf{B}=\left|\begin{array}{cc}
\mathbf{B}_{p} & \mathbf{0} \\
\mathbf{0} & \mathbf{B}_{p}
\end{array}\right|, \quad \mathbf{Q}=\left|\begin{array}{cc}
\mathbf{Q}_{p} & \mathbf{0} \\
\mathbf{0} & \mathbf{Q}_{p}
\end{array}\right|,
$$

where the blocks referring to individual parent hydrocarbons are denoted by subscript $p$. Let us introduce an additional assumption that the new bond(s) emerge between pairs of AOs of subsets $\left\{\chi_{1, I}\right\}$ and $\left\{\chi_{2, I I}\right\}$ (For the case of a single bond, this assumption does not imply any restriction.) As a result, submatrix $\mathbf{W}_{(1)}$ of our first-order Hamiltonian matrix $\mathbf{H}_{(1)}^{(\beta)}$ takes the form

$$
\mathbf{W}_{(1)}=\left|\begin{array}{cc}
\mathbf{0} & \mathbf{K}_{(1)} \\
\mathbf{0} & \mathbf{0}
\end{array}\right|
$$

where $\mathbf{K}_{(1)}$ is a specific nonzero block. For the total matrices $\boldsymbol{\Delta}_{(1)}^{(\beta)}$ and $\boldsymbol{\Gamma}_{(1)}^{(\beta)}$, we accordingly obtain

$$
\begin{aligned}
& \boldsymbol{\Delta}_{(1)}^{(\beta)}=\left|\begin{array}{cc}
\mathbf{0} & \boldsymbol{\Delta}_{(1) p}^{(\beta)} \\
-\boldsymbol{\Delta}_{(1) p}^{(\beta)+} & \mathbf{0}
\end{array}\right|, \\
& \boldsymbol{\Gamma}_{(1)}^{(\beta)}=\left|\begin{array}{cc}
\mathbf{0} & \mathbf{B}_{p} \mathbf{Q}_{p} \boldsymbol{\Delta}_{(1) p}^{(\beta)} \mathbf{Q}_{p} \mathbf{B}_{p}^{+} \\
-\mathbf{B}_{p} \mathbf{Q}_{p} \boldsymbol{\Delta}_{(1) p}^{(\beta)+} \mathbf{Q}_{p} \mathbf{B}_{p}^{+} & \mathbf{0}
\end{array}\right|,
\end{aligned}
$$

where

$$
\boldsymbol{\Delta}_{(1) p}^{(\beta)}=-\int_{0}^{\infty} e^{-\mathbf{Q}_{p} t} \cdot \mathbf{Q}_{p}^{2} \mathbf{B}_{p}^{+} \mathbf{K}_{(1)} \mathbf{Q}_{p} \cdot e^{-\mathbf{Q}_{p} t} d t .
$$

[The solution of Eq. (38) for matrix $\mathbf{W}_{(1)}$ of Eq. (47) is used when deriving Eqs. (48) and (49).] These expressions should be then substituted into Eqs. (37) and (45). As a result, the relevant corrections $\mathbf{P}_{(1)}^{(\beta)}$ and $\mathbf{U}_{(1)}^{(\beta)}$ ' become expressed in terms of submatrices $\mathbf{B}_{p}, \mathbf{Q}_{p}$, and $\boldsymbol{\Delta}_{(1) p}^{(\beta)}$ referring to the parent hydrocarbon.

\section{Analysis of Expressions for Noncanonical MOs of PAHs}

As in Refs. [16, 17], let us define the row matrices $\left(\chi_{1}\right)$ and $\left(\chi_{2}\right)$ containing the subsets of AOs underlying the blocks of the initial Hamiltonian matrix $\mathbf{H}$ of Eqs. (1) and (9), as well as the total row matrix containing $\left(\chi_{1}\right)$ and $\left(\chi_{2}\right)$ as subrows. Passing from the basis of AOs to that of NCMOs may be then described as follows:

$$
\left[\left(\Psi_{1}\right)\left(\Psi_{2}\right)\right]=\left[\left(\chi_{1}\right)\left(\chi_{2}\right)\right] \mathbf{U},
$$

where the transformation matrix $\mathbf{U}$ coincides with that of either Eq. (19) or Eq. (42), and $\left(\Psi_{1}\right)$ and $\left(\Psi_{2}\right)$ are row matrices of occupied and vacant NCMOs, respectively.

Let us begin with the case of the block-diagonal perturbation matrix of Eq. (22). To derive the corresponding NCMOs, the matrix $\mathbf{U}_{(0)}=\mathbf{C}$ and $\mathbf{U}_{(1)}^{(\alpha)}$ defined by Eqs. (6) and (30), respectively, should be substituted into Eq. (50). Therefore, the row matrix $\left(\Psi_{1}^{(\alpha)}\right)$ containing the occupied NCMOs takes the form

$$
\left[\Psi_{1}^{(\alpha)}\right]=\frac{1}{\sqrt{2}}\left\{\left(\chi_{1}\right)\left[\mathbf{I}+\frac{1}{2} \mathbf{X}_{(1)}^{(\alpha)}\right]+\left(\chi_{2}\right)\left[\mathbf{I}+\frac{1}{2} \mathbf{Z}_{(1)}^{(\alpha)}\right] \mathbf{Q B}^{+}\right\}
$$

The particular NCMO $\Psi_{1, i}^{(\alpha)}$ may be then expressed as follows:

$$
\begin{aligned}
& \Psi_{1, i}^{(\alpha)}=\frac{1}{\sqrt{2}}\left(\chi_{1, i}\left[1+\frac{1}{2} \mathbf{X}_{(1) i i}^{(\alpha)}\right]+\frac{1}{2} \sum_{j \neq i)}^{(1)} \chi_{1, j} \mathbf{X}_{(1) j i}^{(\alpha)}\right. \\
& \left.+\sum_{k}^{(2)} \chi_{2, k}\left(\left[1+\frac{1}{2} \mathbf{Z}_{(1) k k}^{(\alpha)}\right]\left(\mathbf{Q B}^{+}\right)_{k i}+\frac{1}{2} \sum_{l(\neq k)}^{(2)} \mathbf{Z}_{(1) k l}^{(\alpha)}\left(\mathbf{Q B}^{+}\right)_{l i}\right\}\right),
\end{aligned}
$$

where the first sum embraces the AOs of the first subset, while the remaining two sums involve the orbitals of the second subset.

A one-to-one correspondence is observed between individual orbitals of subsets $\left(\Psi_{1}^{(\alpha)}\right)$ and $\left(\chi_{1}\right)$ in Eq. (52), i.e., between the occupied $\operatorname{NCMO} \Psi_{1, i}^{(\alpha)}$ and AO $\chi_{1, i}$. Moreover, it is this AO that plays the role of the principal basis function of the given NCMO. The latter conclusion is based on the fact that elements $\left(\mathbf{Q B}^{+}\right)_{k i}$ referring to parent $\mathrm{AHs}$ do 
not exceed 1 [24]. Accordingly, the vacant NCMOs become related to the AOs of the second subset $\left\{\chi_{2}\right\}$. The conclusion that occupied and vacant NCMOs of PAHs are attached to subsets of AOs $\left\{\chi_{1}\right\}$ and $\left\{\chi_{2}\right\}$, respectively, causes no surprise if we recall the analogous relationship for parent AHs underlying the matrix C of Eq. (6) [24]. (An alternative solution of the block-diagonalization problem for the matrix $\mathbf{H}_{(0)}$ of Eq. (1) was also shown to be possible, wherein the subsets $\left\{\chi_{1}\right\}$ and $\left\{\chi_{2}\right\}$ are interchanged, and a somewhat different matrix $\mathbf{C}^{\prime}$ was obtained in this case [24]. Use of this new matrix $\mathbf{C}^{\prime}$ instead of matrix C of Eq. (6) would evidently yield an alternative form of NCMOs of PAHs, wherein the occupied NCMOs are attached to the second subset of AOs.)

The influence of perturbation on the shapes of NCMOs is represented by elements of submatrices $\mathbf{X}_{(1)}^{(\alpha)}$ and $\mathbf{Z}_{(1)}^{(\alpha)}$, as indicated by Eqs. (51) and (52). The same submatrices are also contained within the relevant correction $\mathbf{P}_{(1)}^{(\alpha)}$ of Eq. (27). This implies a certain interrelation between the reshaping of NCMOs of PAHs versus those of parent AHs and the relevant charge and bond order redistribution.

The above-expected relationship is especially evident if we consider the alteration in the extent of localization of the $\mathrm{NCMO} \Psi_{1, i}^{(\alpha)}$ over the respective principal $\mathrm{AO} \chi_{1, i}$. Indeed, the correction of Eq. (52) describing the above-specified alteration proves to be proportional to the population $\left[\mathbf{X}_{(1) i i}^{(\alpha)}\right]$ lost (acquired) by the basis orbital $\chi_{1, i}$ due to perturbation. As a result, the extent of localization of the NCMO $\Psi_{1, i}^{(\alpha)}$ over its principal $\mathrm{AO}$ is predicted to be increased if the latter acquires an additional population, i.e., $\mathbf{X}_{(1) i i}^{(\alpha)}>0$, and vice versa. In other terms, concentration of a population on a specific AO (if any) is shown to be accompanied by an increase of localization of the respective NCMO.

Furthermore, the perturbation under discussion gives rise to emergence of additional delocalization of NCMOs. In particular, the occupied $\mathrm{NCMO} \Psi_{1, i}^{(\alpha)}$ also becomes delocalized over the $\mathrm{AO} \chi_{1, j}$, and the relevant increment is proportional to the respective bond order $\mathbf{X}_{(1) j i}^{(\alpha)}$. As far as the changing contributions of the AOs of the opposite subset are concerned, these are determined by coefficients contained within the curly braces in Eq. (52). It is seen that both the population alterations $\left(\mathbf{Z}_{(1) k k}^{(\alpha)}\right)$ and the changing bond orders $\left(\mathbf{Z}_{(1) k l}^{(\alpha)}\right)$ are present there. Thus, a more involved relation between reshapings of tails of NCMOs over the opposite subset and charge-bond order redistributions may be expected.
For the perturbation $\mathbf{H}_{(1)}^{(\beta)}$ of Eq. (34), the analogue of Eq. (51) takes the form

$$
\left(\Psi_{1}^{(\beta)}\right)=\frac{1}{\sqrt{2}}\left\{\left(\chi_{1}\right) \mathbf{I}+\left(\chi_{2}\right) \mathbf{Q B} \mathbf{B}^{+}\left[\mathbf{I}+\boldsymbol{\Gamma}_{(1)}^{(\beta)}\right]\right\},
$$

where the correction $\mathbf{U}_{(1)}^{(\beta)}$ ' of Eq. (45) is invoked. It is seen that corrections to NCMOs are determined by elements of matrix $\mathbf{Q B}{ }^{+} \boldsymbol{\Gamma}_{(1)}^{(\beta)}$ in this case. Consequently, reshapings of NCMOs of AHs due to perturbation $\mathbf{H}_{(1)}^{(\beta)}$ prove to be related to alterations in bond orders of the intersubset type [see Eq. (37)].

Given that the perturbation matrix $\mathbf{H}_{(1)}^{(\beta)}$ describes the formation of intermolecular bond(s) between two identical initially isolated AHs I and II, we obtain

$$
\left(\Psi_{1}^{(\beta) I}\right)=\frac{1}{\sqrt{2}}\left[\left(\chi_{1}^{I}\right) \mathbf{I}+\left(\chi_{2}^{I}\right) \mathbf{Q}_{p} \mathbf{B}_{p}^{+}-\left(\chi_{2}^{I I}\right) \boldsymbol{\Delta}_{(1) p}^{(\beta)+} \mathbf{Q}_{p} \mathbf{B}_{p}^{+}\right],
$$

where $\left(\Psi_{1}^{(\beta) I}\right)$ contains the reshaped occupied $\mathrm{NCMOs}$ of the first $\mathrm{AH}$ after including it into the final compound. The effect of perturbation is represented by the last term of Eq. (54) determining some increments of AOs from subset $\left\{\chi_{2}^{I I}\right\}$. Hence, the occupied NCMOs of the first parent $\mathrm{AH}$ attached to AOs of the first subset acquire additional tails only over AOs of the opposite $\mathrm{AH}$ belonging to the second subset.

\section{Studies of Individual Molecules as Examples}

\subsection{PYRIDINE MOLECULE}

Let us consider the pyridine molecule in this subsection. Numbering of respective $2 p_{z}$ AOs (see Scheme 1) is chosen to ensure the anti-block-diagonal structure of the relevant Hamiltonian matrix of the parent $\mathrm{AH}$ (benzene) as assumed in Eq. (1). [Nonezero resonance parameters referring to chem-
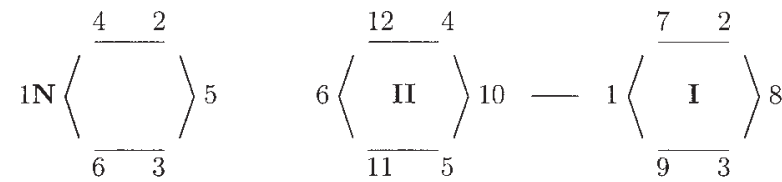

SCHEME 1. Numbering of $2 p_{z}$ AOs of pyridine and biphenyl. 
ical bonds were found in the off-diagonal blocks of the matrix $\mathbf{H}_{(0)}$ in this case.] Passing from benzene to pyridine is modeled by a local perturbation $(\alpha)$ in the Coulomb parameter at the site of substitution, the latter coinciding with the $\mathrm{AO} \chi_{1}$, i.e., $\mathbf{A}_{(1) 11}=\alpha$.

Matrices $\mathbf{B}, \mathbf{Q}$, and $\mathbf{Q B}^{+}$of the parent benzene take the form

$$
\begin{aligned}
& \mathbf{B}=\left|\begin{array}{lll}
1 & 0 & 1 \\
1 & 1 & 0 \\
0 & 1 & 1
\end{array}\right|, \mathbf{Q}=\frac{1}{6}\left|\begin{array}{rrr}
5 & -1 & -1 \\
-1 & 5 & -1 \\
-1 & -1 & 5
\end{array}\right|, \\
& \mathbf{Q B}^{+}=\frac{1}{3}\left|\begin{array}{rrr}
2 & 2 & -1 \\
-1 & 2 & 2 \\
2 & -1 & 2
\end{array}\right| .
\end{aligned}
$$

Matrix $\exp [-\mathbf{Q} t]$ contained within the expression for $\mathbf{Z}_{(1)}^{(\alpha)}$ of Eq. (32) may be easily obtained by diagonalizing matrix $\mathbf{Q}$. Accordingly, for the diagonal and off-diagonal elements of the matrix $\exp [-\mathbf{Q} t]$ we obtain

$$
\begin{aligned}
\{\exp [-\mathbf{Q} t]\}_{i i}=\frac{1}{3}\left[\exp \left(-\frac{1}{2} t\right)+2 \exp (-t)\right] \\
\{\exp [-\mathbf{Q} t]\}_{i j}=\frac{1}{3}\left[\exp \left(-\frac{1}{2} t\right)-\exp (-t)\right]
\end{aligned}
$$

Substituting these expressions into Eq. (32) allows us to derive the following integral formulae for particular elements of matrix $\mathbf{Z}_{(1)}^{(\alpha)}$ :

$$
\begin{aligned}
\mathbf{Z}_{(1) 44}^{(\alpha)}= & \mathbf{Z}_{(1) 66}^{(\alpha)}=\mathbf{Z}_{(1) 46}^{(\alpha)}=-\frac{\alpha}{36} \\
& \int_{0}^{\infty}\left[\exp \left(-\frac{1}{2} t\right)+2 \exp (-t)\right]^{2} d t \\
\mathbf{Z}_{(1) 45}^{(\alpha)}= & \mathbf{Z}_{(1) 56}^{(\alpha)}=-\frac{\alpha}{36} \int_{0}^{\infty}\left[\exp \left(-\frac{1}{2} t\right)-4 \exp (-t)\right] \\
& \times\left[\exp \left(-\frac{1}{2} t\right)+2 \exp (-t)\right] d t, \mathbf{Z}_{(1) 55}^{(\alpha)} \\
= & -\frac{\alpha}{36} \int_{0}^{\infty}\left[\exp \left(-\frac{1}{2} t\right)-4 \exp (-t)\right]^{2} d t,
\end{aligned}
$$

and the final matrices $\mathbf{Z}_{(1)}^{(\alpha)}$ and $\mathbf{X}_{(1)}^{(\alpha)}$ are

$$
\begin{gathered}
\mathbf{Z}_{(1)}^{(\alpha)}=-\frac{\alpha}{108}\left|\begin{array}{rrr}
17 & -13 & 17 \\
-13 & 11 & -13 \\
17 & -13 & 17
\end{array}\right|, \\
\mathbf{X}_{(1)}^{(\alpha)}=\frac{\alpha}{108}\left|\begin{array}{rrr}
43 & -5 & -5 \\
-5 & 1 & 1 \\
-5 & 1 & 1
\end{array}\right|,
\end{gathered}
$$

where Eq. (18) is used to obtain the second matrix. The orbital-orbital polarizabilities of benzene may be obtained from elements of these matrices after taking the relevant derivatives with respect to parameter $\alpha$, as shown in Eq. (21), and the relevant numerical values coincide with those obtained by traditional methods [30]. At the same time, Eq. (57) yields a new integral representation of these polarizabilities.

Let us turn now to the NCMOs of pyridine following from Eq. (52) after substituting Eqs. (55) and (58). Thus, the occupied $\operatorname{NCMO} \Psi_{1,1}$ attached to the site of substitution, takes the form

$$
\begin{aligned}
\Psi_{1,1}= & \frac{1}{\sqrt{2}}\left\{\left[\chi_{1}+\frac{2}{3}\left(\chi_{4}+\chi_{6}\right)-\frac{1}{3} \chi_{5}\right]+\alpha\left[\frac{43}{216} \chi_{1}\right.\right. \\
& \left.\left.-\frac{5}{216}\left(\chi_{2}+\chi_{3}\right)-\frac{27}{216}\left(\chi_{4}+\chi_{6}\right)+\frac{21}{216} \chi_{5}\right]\right\},
\end{aligned}
$$

where the first square brackets contain the zeroorder terms referring to benzene [24], while the second ones involve the corrections due to substitution. It is seen that the extent of localization of the $\operatorname{NCMO} \Psi_{1,1}$ over the principal AO $\chi_{1}$ is increased in accordance with the increased occupation number of this basis orbital due to perturbation. Thus, reshaping of the $\mathrm{NCMO} \Psi_{1,1}$ reflects the trend toward formation of a lone pair orbital belonging to the nitrogen atom. Furthermore, negative increments referring to meta positions 2 and 3 with respect to the heteroatom arise in the perturbed NCMO. The most important feature of the orbital $\Psi_{1,1}$, however, consists of reduction of absolute values of increments corresponding to both ortho- and para-positions with respect to heteroatom. Consequently, a certain parallelism is observed between reshaping of the NCMO $\Psi_{1,1}$ and the rule of the alternating polarity; viz. withdrawal of population from the ortho/para-positions is accompanied by reduction of contribution of respective AOs in the NCMO, whereas emergence of additional population at the meta position is accordingly accompanied by new increments within the same NCMO. Certain comments of this conclusion should be added. From Eq. (58) it is seen that all the elements of matrix $\mathbf{Z}_{(1)}^{(\alpha)}$ of benzene have comparable absolute values. The same refers also to elements of the matrix $\mathrm{QB}^{+}$of Eq. (55). This implies that the alterations in the intersubset delocalization of NCMOs are conditioned not only by occupation numbers $\left[\mathbf{Z}_{(1) k k}^{(\alpha)}\right]$ of 
AOs of the second subset, but also by bond orders between the latter $\left[\mathbf{Z}_{(1) k l}^{(\alpha)}\right]$. In this context, the aboveobserved parallelism between the reshaping pattern of the NCMO $\Psi_{1,1}$ and alterations in the populations of AOs is even more surprising.

Let us now consider the remaining occupied NCMOs of pyridine. Thus, the orbital $\Psi_{1,2}$ attached to the $\mathrm{AO} \chi_{2}$ takes the form

$$
\begin{aligned}
\Psi_{1,2} & =\frac{1}{\sqrt{2}}\left\{\left[\chi_{2}+\frac{2}{3}\left(\chi_{4}+\chi_{5}\right)-\frac{1}{3} \chi_{6}\right]+\alpha\left[\frac{1}{216} \chi_{2}\right.\right. \\
- & \left.\left.\frac{5}{216} \chi_{1}+\frac{1}{216} \chi_{3}+\frac{3}{216}\left(\chi_{4}-\chi_{5}\right)+\frac{3}{216} \chi_{6}\right]\right\} .
\end{aligned}
$$

It is seen that the extent of localization of this NCMO over the principal $\mathrm{AO} \chi_{2}$ is also increased due to perturbation. The absolute value of this alteration, however, is considerably smaller as compared with that referring to the $\mathrm{NCMO} \Psi_{1,1}$ of Eq. (59). This result is in line with relative values of the corresponding population alterations. The remaining corrections of Eq. (60) give rise to a certain desymmetrization of the $\mathrm{NCMO} \Psi_{1,2}$. In particular, dissimilar contributions of AOs $\chi_{1}$ and $\chi_{3}$ may be mentioned, where a larger absolute value of the increment corresponds to the site of substitution. Moreover, the total increments of AOs $\chi_{4}$ and $\chi_{5}$ also become dissimilar in the final $\mathrm{NCMO} \Psi_{1,2}$ owing to different signs of the relevant first-order corrections, i.e., the contribution of the $\mathrm{AO} \chi_{4}$ proves to be increased, whereas that of the $\mathrm{AO} \chi_{5}$ is reduced compared with the respective values for the parent molecule. Thus, the reshaping pattern of the $\operatorname{NCMO} \Psi_{1,2}$ is such that a trend toward the formation of a double bond between AOs $\chi_{2}$ and $\chi_{4}$ is observed along with weakening of the bond $\mathrm{C}_{2}-\mathrm{C}_{5}$. This result is in line with the emergence of a certain contribution of the para-quinoidal structure for pyridine.

Comparison of Eqs. (59) and (60), in turn, demonstrates that the total extent of reshaping of the $\operatorname{NCMO} \Psi_{1,2}$ is less significant relative to that of $\Psi_{1,1}$. In other terms, it is the NCMO $\Psi_{1,1}$ that is influenced by the given perturbation most substantially. That is why the NCMO $\Psi_{1,1}$ is primarily responsible for the relevant charge redistribution. Just this fact may be considered as the origin of the abovediscussed parallelism between the reshaping pattern of this NCMO and the population alterations due to perturbation.

\subsection{BIPHENYL MOLECULE}

Numbering of $2 p_{z}$ AOs of carbon atoms of biphenyl may be chosen so that the matrices $\mathbf{B}_{p}$ and $\mathbf{Q}_{p}$ of Eq. (46) coincide with those of Eq. (55) (see Scheme 1). The same then also refers to the matrix $\exp \left[-\mathbf{Q}_{p} t\right]$, which coincides with the $\exp [-\mathbf{Q} t]$ defined by Eq. (56).

Let the only nonzero element of submatrix $\mathbf{K}_{(1)}$ of Eq. (47) referring to the new bond $C_{1}-C_{10}$ to be denoted by $\gamma$. As a result, the matrix $\Delta_{(1) p}^{(\beta)}$ defined by Eq. (49) and contained within the expression for NCMOs of Eq. (54) takes the form

$$
\Delta_{(1) p}^{(\beta)}=\frac{\gamma}{36}\left|\begin{array}{rrr}
-9 & 1 & 1 \\
7 & -1 & -1 \\
-9 & 1 & 1
\end{array}\right| .
$$

Consequently, the NCMOs of biphenyl $\Psi_{1,1}$ and $\Psi_{1,2}$ attached to the AOs $\chi_{1}$ and $\chi_{2}$, respectively, may be expressed as follows:

$$
\begin{aligned}
& \Psi_{1,1}=\frac{1}{\sqrt{2}}\{ {\left[\chi_{1}+\frac{2}{3}\left(\chi_{7}+\chi_{9}\right)-\frac{1}{3} \chi_{8}\right] } \\
&\left.+\gamma\left[\frac{43}{108} \chi_{10}-\frac{5}{108}\left(\chi_{11}+\chi_{12}\right)\right]\right\}, \\
& \Psi_{1,2}=\frac{1}{\sqrt{2}}\left\{\left[\chi_{2}+\frac{2}{3}\left(\chi_{7}+\chi_{8}\right)-\frac{1}{3} \chi_{9}\right]\right. \\
&\left.+\gamma\left[-\frac{5}{108} \chi_{10}+\frac{1}{108}\left(\chi_{11}+\chi_{12}\right)\right]\right\} .
\end{aligned}
$$

It is seen that $\operatorname{NCMOs} \Psi_{1,1}$ and $\Psi_{1,2}$ acquire additional tails localized on the second ring after emergence of the new bond $\mathrm{C}_{1}-\mathrm{C}_{10}$. Moreover, the most important new increments of these tails are localized at the site of perturbation, i.e., at $\mathrm{AO} \chi_{10}$. Comparison of total extents of additional tails for NCMOs $\Psi_{1,1}$ and $\Psi_{1,2}$, in turn, shows that it is the NCMO $\Psi_{1,1}$ that is reshaped more significantly. This fact reflects a trend toward formation of a four-center NCMO instead of a three-center NCMO after emergence of the new bond. This result causes no surprise if we recall the dependence between the shapes of NCMOs of AHs and the numbers of the nearest neighbors for the respective principal AOs established previously [24]. 


\section{Summary}

Two points may be mentioned as the principal achievements of the above study. The first point concerns reinterpretation of the classical results about charge and bond order redistributions in AHs due to perturbation as a part of the noncanonical theory of MOs along with supplementing them with the following contributions: (i) derivation of algebraic expressions for the charge-bond order matrix of PAHs in terms of entire blocks of the common Hamiltonian matrix of parent hydrocarbons that indicate the decisive role of molecular topology in the formation of charge and bond order distribution; and (ii) suggestion of a new integral representation for orbital-orbital polarizabilities of $\mathrm{AHs}$ that is an alternative to the classical one.

The second point consists of the development of the remaining part of the noncanonical theory of MOs for PAHs embracing the application of NCMOs as an alternative localized way of representing electronic structures. The principal steps of this development are: (i) formulation and solution of the block-diagonalization problem for PAHs originating from the Brillouin theorem and determining the NCMOs of these molecules; (ii) derivation of expressions for the common NCMO representation matrix of PAHs in terms of entire blocks of the relevant charge-bond order matrix; (iii) interpretation of charge redistribution in $\mathrm{AHs}$ due to perturbation in terms of reshaped NCMOs and vice versa; and (iv) analysis of NCMOs of individual molecules (i.e., of pyridine and biphenyl) and establishing of the interdependence between the initial shape of the given NCMO and the extent of its subsequent reshaping due to perturbation.

The contribution of the results obtained to the theory of electronic structures of molecules in general may be summarized as follows:

1. The results contribute to development of direct ways of obtaining NCMOs of molecules without invoking CMOs. Moreover, the power series for NCMO representation matrices derived in this paper allow us to relate the NCMOs of perturbed systems to those of parent molecules without invoking of additional localization criteria.

2. The results demonstrate possibilities of the NCMO method in reflecting chemical structures of molecules and their alterations when passing from one class of compounds to another. Additional support for this conclusion consists of the fact that changes in shapes of NCMOs predicted by this method are in line with those resulting from the simple resonance theory.

3. The solution of the block-diagonalization problem for the Hamiltonian matrix of PAHs supplements the noncommutative RSPT [16] with the case of two quasi-degenerate subsets of basis functions. Moreover, the solution suggested is likely to present the first nontrivial employment of the most general version of the NCRSPT corresponding to the zero-order matrix of a block-diagonal constitution.

\section{References}

1. March, J. Advanced Organic Chemistry, Reactions, Mechanisms and Structure; Wiley-Interscience: New York, 1985.

2. Becker, H. G. O. Einfürung in die Elektronentheorie Organisch Chemischen Reaktionen; Deutscher Verlag der Wissenschaften: Berlin, 1974.

3. Dewar, M. J. S.; Dougherty, R. C. The PMO Theory of Organic Chemistry; Plenum: New York, 1975.

4. Zahradnik, R.; Polak, R. Elements of Quantum Chemistry; Plenum: New York, 1980.

5. Tedder, J. M.; Nechvatal, A. Pictorial Orbital Theory; Pitman: London, 1985.

6. Zülicke, L. Quantenchemie, Vol. 1, Grundlagen and Algemeine Methoden; VEB Deutscher Verlag der Wissenschaften: Berlin, 1973.

7. Huzinaga, S. The MO Method; Mir: Moscow, 1983 [in Russian].

8. Chalvet, O., Ed. Localization and Delocalization in Quantum Chemistry, Atoms and Molecules in the Ground State, Vol. 1, Reidel; Dordrecht, 1975.

9. Daudey, J. P. Chem Phys Lett 1974, 24, 574.

10. Mayer, I. Chem Phys Lett 1982, 89, 390.

11. Surjan, P. R.; Mayer, I.; Kertesz, M. J Chem Phys 1982, 77, 2454.

12. Mayer, I.; Surjan, P. R. J Chem Phys 1984, 80, 5649.

13. Gineityte, V. J Mol Struct (Theochem) 1993, 288, 111.

14. Gineityte, V. J Mol Struct (Theochem) 1995, 333, 297.

15. Gineityte, V. J Mol Struct (Theochem) 1995, 343, 183.

16. Gineityte, V. Int J Quantum Chem 1998, 68, 119.

17. Gineityte, V. Int J Quantum Chem 1999, 72, 559.

18. Gineityte, V. Lithuanian J Phys 2004, 44, 219.

19. Gineityte, V. Int J Quantum Chem 2000, 77, 534

20. Gineityte, V. J Mol Struct (Theochem) 1996, 364, 85.

21. Gineityte, V. J Mol Struct (Theochem) 1998, 430, 97.

22. Gineityte, V. J Mol Struct (Theochem) 1999, 487, 231.

23. Segal, G. A., Ed. Semiempirical Methods of Electronic Struc- 


\section{LMOs OF PERTURBED ALTERNANT HYDROCARBONS}

ture Calculations, Part A: Techniques; Plenum: New York, 1977.

24. Gineityte, V. Int J Quantum Chem 2005, 101, 274.

25. Coulson, C. A.; Longuet-Higgins, H. C. Proc R Soc (London) 1947, A191, 39.

26. Coulson, C. A.; Longuet-Higgins, H. C. Proc R Soc (London) 1947, A192, 16.

27. Coulson, C. A.; Longuet-Higgins, H. C. Proc R Soc (London) 1948, A193, 447, 456.

28. Coulson, C. A.; Longuet-Higgins, H. C. Proc R Soc (London) 1948, A195, 188.
29. Dewar, M. J. S. The Molecular Orbital Theory of Organic Chemistry; McGraw-Hill: New York, 1969.

30. Basilevskii, M. V. Metod Molekuliarnych Orbit i Reaktsionnaya Sposobnost Organitcheskich Molekul; Khimia: Moscow, 1969 [in Russian].

31. Mestechkin, M. M. Metod Matricy Plotnosti v Teorii Molekul; Naukova Dumka: Kiev, 1977 [in Russian].

32. Gutman, I. Z Naturforsch 1981, 36A, 1112.

33. Hall, G. G. Proc R Soc (London) 1955, A229, 251.

34. Lankaster, P. Theory of Matrices; Academic Press: New York, 1969. 